\title{
Perfect Packings in Quasirandom Hypergraphs II
}

\author{
John Lenz * \\ University of Illinois at Chicago \\ lenz@math.uic.edu
}

\author{
Dhruv Mubayi ${ }^{\dagger}$ \\ University of Illinois at Chicago \\ mubayi@math.uic.edu
}

October 16, 2018

\begin{abstract}
For each of the notions of hypergraph quasirandomness that have been studied, we identify a large class of hypergraphs $F$ so that every quasirandom hypergraph $H$ admits a perfect $F$-packing. An informal statement of a special case of our general result for 3-uniform hypergraphs is as follows. Fix an integer $r \geq 4$ and $0<p<1$. Suppose that $H$ is an $n$-vertex triple system with $r \mid n$ and the following two properties:

- for every graph $G$ with $V(G)=V(H)$, at least $p$ proportion of the triangles in $G$ are also edges of $H$,

- for every vertex $x$ of $H$, the link graph of $x$ is a quasirandom graph with density at least $p$.

Then $H$ has a perfect $K_{r}^{(3)}$-packing. Moreover, we show that neither hypotheses above can be weakened, so in this sense our result is tight. A similar conclusion for this special case can be proved by Keevash's hypergraph blowup lemma, with a slightly stronger hypothesis on $H$.
\end{abstract}

\section{Introduction}

A $k$-uniform hypergraph $H$ ( $k$-graph for short) is a collection of $k$-element subsets (edges) of a vertex set $V(H)$. For a $k$-graph $H$ and a subset $S$ of vertices of size at most $k-1$, define the $(k-|S|)$-graph $N_{H}(S):=\{T \subseteq V(H)-S: T \cup S \in H\}$. Also, let $d_{H}(S)=\left|N_{H}(S)\right|$. When $S=\{x\}$, we write $N_{H}(x)$ and $d_{H}(x)$. The minimum $\ell$-degree of $H$, written $\delta_{\ell}(H)$, is the minimum of $d_{H}(S)$ taken over all $\ell$-sets $S \in\left(\begin{array}{c}V(H) \\ \ell\end{array}\right)$. The minimum codegree of $H$ is $\delta_{k-1}(H)$ and the minimum degree is $\delta(H)=\delta_{1}(H)$. The complete $k$-graph on $r$ vertices, denoted $K_{r}^{(k)}$ (or sometimes just $\left.K_{r}\right)$ is the $k$-graph with vertex set $[r]$ and all $\left(\begin{array}{l}r \\ k\end{array}\right)$ edges.

${ }^{*}$ Research partly supported by NSA Grant H98230-13-1-0224.

${ }^{\dagger}$ Research supported in part by NSF Grants 0969092 and 1300138. 
If $H$ is a $k$-graph and $x \in V(H)$, the link of $x$, written $L_{H}(x)$, is the $(k-1)$-graph whose vertex set is $V(H)-\{x\}$ and whose edge set is $N_{H}(x)$. We write $v(H)$ for $|V(H)|$.

Let $G$ and $F$ be $k$-graphs. We say that $G$ has a perfect $F$-packing if the vertex set of $G$ can be partitioned into copies of $F$. Minimum degree conditions that force perfect $F$ packings in graphs have a long history and have been well studied [1, 11, 21, 23. In the past decade there has been substantial interest in extending these result to $k$-graphs [9, 12, 15, 16, 17, 22, 24, 25, 30, 31, 32, 33, 34, 39, 40. Despite this activity many basic questions in this area remain open. For example, for $k \geq 5$ the minimum degree threshold which forces a perfect matching in $k$-graphs is not known.

A key ingredient in the proofs of most of the previously cited results are specially designed random-like or quasirandom properties of $k$-graphs that imply the existence of perfect $F$ packings. There is a rather well-defined notion of quasirandomness for graphs that originated in early work of Thomason [36, 37] and Chung-Graham-Wilson [7]. These graph quasirandom properties, when generalized to $k$-graphs, provide a rich structure of inequivalent hypergraph quasirandom properties (see [29, 38]). In [28], the authors studied in detail the packing problem for the simplest of these quasirandom properties, the so-called weak hypergraph quasirandomness. A hypergraph is linear if every two edges share at most one vertex. Results of [28] showed that weak hypergraph quasirandomness and an obvious minimum degree condition suffices to obtain perfect $F$-packings for all linear $F$, but the result does not hold for certain $F$ that are very close to being linear.

In this paper, we address the packing problem for the other quasirandom properties. A special case of our result identifies what hypergraph quasirandom property and what condition on the link of each vertex is required in order to be able to guarantee a perfect $K_{r}^{(k)}$-packing for all $r$ (which implies a perfect $F$-packing for all $F$ ). The quasirandom property naturally has great resemblance to those used in the various (strong) hypergraph regularity lemmas. Keevash's hypergraph blowup lemma [14] has as a corollary that the super-regularity of complexes implies the existence of perfect packings, but our main result below (Theorem 11) shows that a weaker notion of quasirandomness is enough to obtain perfect packings of complete hypergraphs. In fact, we are able to do more: for many of the hypergraph quasirandom properties that have been studied previously in the literature, we give a class of hypergraphs $F$ for which we can find a perfect packing. Before stating Theorem 1, we need to define these notions of hypergraph quasirandomness.

\section{$1.1 \quad$ Notions of Hypergraph Quasirandomness}

Our definitions are closely related to the definitions by Towsner [38], which gives the most general treatment of hypergraph quasirandomness.

Definition. Let $X$ be a finite set and let $2^{X}=\{A: A \subseteq X\}$. An antichain is an $\mathcal{I} \subseteq 2^{X}$ such that $A \subsetneq B$ for all $A, B \in \mathcal{I}$. A full antichain is an antichain $\mathcal{I} \subseteq 2^{X}$ such that $|\mathcal{I}| \geq 2$ and for all $x \in X$, there exists $I \in \mathcal{I}$ with $x \in I$.

Definition. Let $k \geq 1$, let $\mathcal{I} \subseteq 2^{[k]}$ be an antichain, and let $H$ be a $k$-graph. An $\mathcal{I}$-layout in $H$ is a tuple of uniform hypergraphs $\Lambda=\left(\lambda_{I}\right)_{I \in \mathcal{I}}$ where $\lambda_{I}$ is an $|I|$-uniform hypergraph 
on vertex set $V(H)$. If $\Lambda$ is an $\mathcal{I}$-layout, then the $k$-cliques of $\Lambda$, denoted $K_{k}(\Lambda)$, is the set of all vertex tuples $\left(x_{1}, \ldots, x_{k}\right)$ such that $x_{1}, \ldots, x_{k}$ are distinct vertices and for each $I \in \mathcal{I},\left\{x_{i}: i \in I\right\} \in \lambda_{I}$. In an abuse of notation, we will denote by $H \cap K_{k}(\Lambda)$ the $k$-tuples $\left(x_{1}, \ldots, x_{k}\right)$ such that $\left(x_{1}, \ldots, x_{k}\right) \in K_{k}(\Lambda)$ and $\left\{x_{1}, \ldots, x_{k}\right\} \in H$.

We now are ready to define hypergraph quasirandomness.

Definition. Let $0<\mu, p<1$. A k-graph $H$ satisfies $\operatorname{Disc}^{(k)}(\mathcal{I}, \geq p, \mu)$ if for every $\mathcal{I}$-layout $\Lambda$,

$$
\left|H \cap K_{k}(\Lambda)\right| \geq p\left|K_{k}(\Lambda)\right|-\mu n^{k} .
$$

The stronger property $\operatorname{Disc}^{(k)}(\mathcal{I}, p, \mu)$ stipulates that for every $\mathcal{I}$-layout $\Lambda$,

$$
|| H \cap K_{k}(\Lambda)|-p| K_{k}(\Lambda)|| \leq \mu n^{k} .
$$

Example. Let $k=3$ and $\mathcal{I}=\{\{1,2\},\{2,3\}\}$. A 3-graph $H$ satisfies $\operatorname{Disc}^{(3)}(\mathcal{I}, \geq p, \mu)$ if for every two graphs $\lambda_{12}$ and $\lambda_{23}$ with vertex set $V(H)$, the number of tuples $(x, y, z)$ with $\{x, y, z\} \in H, x y \in \lambda_{12}$, and $y z \in \lambda_{23}$ is at least $p\left|K_{3}\left(\lambda_{12}, \lambda_{23}\right)\right|-\mu n^{3}$, where $K_{3}\left(\lambda_{12}, \lambda_{23}\right)$ is the set of tuples $(x, y, z)$ with $x y \in \lambda_{12}$ and $y z \in \lambda_{23}$.

Several special cases of this definition deserve mention, since essentially all previously studied hypergraph quasirandomness properties are related to $\operatorname{Disc}^{(k)}(\mathcal{I}, \geq p, \mu)$ for some $\mathcal{I}$.

- When $\mathcal{I}=\{\{1\}, \ldots,\{k\}\}$, then $\operatorname{Disc}^{(k)}(\mathcal{I}, \geq p, \mu)$ is exactly the property $\left(p, \frac{\mu}{k !}\right)$-dense from [28] and is closely related to weak quasirandomness studied in [8, 10, 18, 35].

- More generally, when $\mathcal{I}$ is a partition the property $\operatorname{Disc}^{(k)}(\mathcal{I}, p, \mu)$ is essentially the property Expand [ $\pi]$ studied in [26, 27, 29]. In particular, when $\mathcal{I}=\{\{1, \ldots, k-$ $1\},\{k\}\}$, then $\operatorname{Disc}^{(k)}(\mathcal{I}, \geq p, \mu)$ is essentially equivalent to the property considered recently by Keevash (the property called "typical" in [13]) in his recent proof of the existence of designs.

- When $\mathcal{I}=\left(\begin{array}{c}{[k]} \\ \ell\end{array}\right)$, then $\operatorname{Disc}^{(k)}(\mathcal{I}, p, \mu)$ is closely related to the property CliqueDisc $[\ell]$ studied in [2, 3, 4, 5, 6, 19, 29].

- When $\mathcal{I}=\left\{I \in\left(\begin{array}{c}{[k]} \\ k-1\end{array}\right):\{1, \ldots, \ell\} \subseteq I\right\}$, then $\operatorname{Disc}^{(k)}(\mathcal{I}, p, \mu)$ is essentially the same as the property Deviation $[\ell]$ studied in [4, 5, 3, 19, 29].

- Finally, note that $\operatorname{Disc}^{(k)}(\{\emptyset\}, \geq p, \mu)$ is equivalent to $|H| \geq p\left(\begin{array}{c}v(H) \\ k\end{array}\right)-\frac{\mu}{k !} n^{k}$, since $K_{k}(\{\emptyset\})$ is the set of all ordered $k$-tuples of distinct vertices.

Definition. Let $\mathcal{I} \subseteq 2^{[k]}$ be an antichain. A k-graph $F$ is $\mathcal{I}$-adapted if there exists an ordering $E_{1}, \ldots, E_{m}$ of the edges of $F$ and bijections $\phi_{i}: E_{i} \rightarrow[k]$ such that for each $1 \leq j<i \leq m$, the following holds: there exists an $I \in \mathcal{I}$ with $\left\{\phi_{i}(x): x \in E_{j} \cap E_{i}\right\} \subseteq I \in \mathcal{I}$. 
In words, $F$ is $\mathcal{I}$-adapted if the set of labels assigned to $E_{i}$ which appear on $E_{j} \cap E_{i}$ is a subset of a set in $\mathcal{I}$.

Let $\mathcal{I} \subseteq 2^{[k]}$ and $\mathcal{J} \subseteq 2^{[k-1]}$ be antichains. A $k$-graph $F$ is $(\mathcal{I}, \mathcal{J})$-adapted if $F$ is $\mathcal{I}$ adapted and there exists $x \in V(F)$, an ordering $E_{1}, \ldots, E_{m}$ of the edges of $F$, and bijections $\psi_{i}: E_{i} \rightarrow[k]$ such that for all $1 \leq j<i \leq m$, the following holds.

- If $x \notin E_{i}$ then there exists $I \in \mathcal{I}$ with $\left\{\psi_{i}(y): y \in E_{j} \cap E_{i}\right\} \subseteq I$.

- If $x \in E_{i}$ then $\psi_{i}(x)=k$ and there exists $J \in \mathcal{J}$ with $\left\{\psi_{i}(y): y \in E_{j} \cap E_{i}, y \neq x\right\} \subseteq J$.

\subsection{Our Results}

The following is our main result.

Theorem 1. Let $k \geq 2, \mathcal{I} \subseteq 2^{[k]}$ be a full antichain, $\mathcal{J} \subseteq 2^{[k-1]}$, and $0<\alpha, p<1$. For every $(\mathcal{I}, \mathcal{J})$-adapted $k$-graph $F$, there exists $\mu>0$ and $n_{0}$ so that the following holds. Let $H$ be an $n$-vertex $k$-graph where $n \geq n_{0}$ and $v(F) \mid n$. Suppose that $H$ satisfies $\operatorname{Disc}^{(k)}(\mathcal{I}, \geq p, \mu)$ and that $L_{H}(x)$ satisfies $D i s c^{(k-1)}(\mathcal{J}, \geq \alpha, \mu)$ for all $x \in V(H)$. Then $H$ has a perfect $F$-packing.

It is straightforward to see that if $\mathcal{I}$ and $\mathcal{I}^{\prime}$ are such that for every $I^{\prime} \in \mathcal{I}^{\prime}$, there exists $I \in \mathcal{I}$ with $I^{\prime} \subseteq I$, then $\operatorname{Disc}^{(k)}(\mathcal{I}, \geq p, \mu) \Rightarrow \operatorname{Disc}^{(k)}\left(\mathcal{I}^{\prime}, \geq p, \mu\right)$. Also, if $\mathcal{I}=\left(\begin{array}{c}{[k]} \\ k-1\end{array}\right)$ and $\mathcal{J}=\left(\begin{array}{c}{[k-1]} \\ k-2\end{array}\right)$, then every $F$ is $(\mathcal{I}, \mathcal{J})$-adapted. Thus to find the weakest quasirandom condition to apply Theorem 1 to a given $k$-graph $F$, one should find the minimal $\mathcal{I}$ and $\mathcal{J}$ for which $F$ is $(\mathcal{I}, \mathcal{J})$-adapted. For example, if $C=\{a b c, b c d$, def, aef $\}$, then $C$ is $(\mathcal{I}, \mathcal{J})$-adapted where $\mathcal{I}=\{\{1,2\},\{3\}\}$ and $\mathcal{J}=\{\emptyset\}$ (let $x=a$ and order the edges which contain $a$ first).

As mentioned above, special cases of $\operatorname{Disc}^{(k)}(\mathcal{I}, \geq p, \mu)$ correspond to previously studied quasirandom properties so that Theorem 1 generalizes several previous results.

- Let $k=2$. The only full antichain is $\mathcal{I}=\{\{1\},\{2\}\}$. For this $\mathcal{I}$, all graphs $F$ are $(\mathcal{I}, \mathcal{J})$ adapted if $\mathcal{J}=\{\emptyset\}$. To see this, pick $x \in V(F)$ and place all edges incident to $x$ first in the ordering for the definition of $(\mathcal{I}, \mathcal{J})$-adapted. Now the property $\operatorname{Disc}^{(2)}(\mathcal{I}, \geq p, \mu)$ just states that $G$ is quasirandom (in fact only "one-sided" quasirandom). Also, the condition " $L_{H}(x)$ satisfies $\operatorname{Disc}^{(1)}(\{\emptyset\}, \geq \alpha, \mu)$ for every $x \in V(H)$ " is equivalent to the condition that $\delta(H) \geq(\alpha-\mu)(n-1)$. To see this, recall from before that if $H^{\prime}$ is an $r$-graph the property " $H^{\prime}$ satisfies $\operatorname{Disc}^{(1)}(\{\emptyset\}, \geq \alpha, \mu)$ is equivalent to the property that $\left|H^{\prime}\right| \geq \alpha\left(\begin{array}{c}v\left(H^{\prime}\right) \\ r\end{array}\right)-\frac{\mu}{r !} v\left(H^{\prime}\right)^{r}$. Thus Theorem 1 for $k=2$ states that if $G$ is an $n$-vertex quasirandom graph, $v(F) \mid n$, and $\delta(G) \geq(\alpha-\mu)(n-1)$, then $G$ has a perfect $F$-packing. This fact is a simple consequence of the blowup lemma of Komlós-SárközySzemerédi [20].

- For $k \geq 2$ with $\mathcal{I}$ a partition into singletons, we obtain exactly [28, Theorem 3]. In this case, $\operatorname{Disc}^{(k)}(\mathcal{I}, \geq p, \mu)$ is equivalent to $\left(p, \frac{\mu}{k !}\right)$-dense from [28], an $\mathcal{I}$-adapted $k$-graph is a linear $k$-graph, and one can take $\mathcal{J}=\{\emptyset\}$. Similar to the previous paragraph, the condition " $L_{H}(x)$ satisfies $\operatorname{Disc}^{(k-1)}(\{\emptyset\}, \geq \alpha, \mu)$ for every $x \in V(H)$ " is equivalent to the condition that $\delta(H) \geq \alpha\left(\begin{array}{c}v(H)-1 \\ k-1\end{array}\right)-\frac{\mu}{(k-1) !} v(H)^{k-1}$. 
- If $\mathcal{I}=\left(\begin{array}{c}{[k]} \\ k-1\end{array}\right)$ and $\mathcal{J}=\left(\begin{array}{c}{[k-1]} \\ k-2\end{array}\right)$ then every $k$-graph $F$ is $(\mathcal{I}, \mathcal{J})$-adapted. Thus Theorem 1 implies the following corollary.

Corollary 2. Fix $2 \leq k \leq r$. For every $0<\alpha, p<1$, there exists $\mu>0$ and $n_{0}$ such that the following holds. Let $H$ be an $n$-vertex $k$-graph with $n \geq n_{0}$ and $r \mid n$. If $H$ satisfies Disc ${ }^{(k)}\left(\left(\begin{array}{c}{[k]} \\ k-1\end{array}\right), \geq p, \mu\right)$ and $L_{H}(x)$ satisfies Disc ${ }^{(k-1)}\left(\left(\begin{array}{c}{[k-1]} \\ k-2\end{array}\right), \geq \alpha, \mu\right)$ for every $x \in V(H)$, then $H$ has a perfect $K_{r}^{(k)}$-packing.

Keevash's hypergraph blowup lemma [14] also guarantees perfect $K_{r}^{(k)}$-packings under certain regularity conditions, however the hypotheses of Corollary 2 are slightly weaker. Indeed, the main extra requirement that [14] places on $H$ is [14, Definition 3.16 part (iii)]; translated into our language, for 3-graphs this property says roughly that for every $x \in V(H)$, if $W$ is a set of triples where each triple contains some pair from $L_{H}(x)$, then $|H \cap W| \approx p|W|$.

Next, we investigate if either of the conditions $\operatorname{Disc}^{(k)}(\mathcal{I}, \geq p, \mu)$ or $\operatorname{Disc}^{(k-1)}(\mathcal{J}, \geq \alpha, \mu)$ in the links from Theorem 1 can be weakened. This question was studied by the authors [28] in detail when $\mathcal{I}$ is a partition, and it turns out that for certain non-linear $F$ it is possible to weaken the conditions (see [28] for details). Most likely, the constructions and results from [28] can be generalized to all $\mathcal{I}$. In this paper, we focus only on the case $\mathcal{I}=\left(\begin{array}{c}{[k]} \\ k-1\end{array}\right)$ and $\mathcal{J}=\left(\begin{array}{c}{[k-1]} \\ k-2\end{array}\right)$, which corresponds to the condition required for perfect $K_{r}^{(k)}$-packings. In this case, neither condition can be weakened, so that Theorem 1 cannot be improved in general.

Proposition 3. For every $k \geq 3$ there exists an $r$ (depending only on $k$ ) such that the following holds. Let $\alpha=p=\frac{k-1}{k}$ and let $\mathcal{I} \subseteq 2^{[k]}$ be a full antichain where $\mathcal{I} \neq\left(\begin{array}{c}{ }^{[k]} \\ k-1\end{array}\right)$. For every $\mu>0$, there exists $n_{0}$ such that for all $n \geq n_{0}$ there exists an $n$-vertex $k$-graph $H$ which

- satisfies $\operatorname{Disc}^{(k)}(\mathcal{I}, \geq p, \mu)$,

- fails $\operatorname{Disc}^{(k)}\left(\left(\begin{array}{c}{[k]} \\ k-1\end{array}\right), \geq p, \mu\right)$,

- for every $x \in V(H)$ the link $L_{H}(x)$ satisfies $\operatorname{Disc}(k-1)\left(\left(\begin{array}{c}k-1] \\ k-2\end{array}\right), \geq \alpha, \mu\right)$,

- has no copy of $K_{r}$ (so no perfect $K_{r}$-packing).

Proposition 4. For every $k \geq 3$ there exists an $r$ (depending only on $k$ ) such that the following holds. Let $\alpha=p=\frac{k-1}{k}$ and let $\mathcal{J} \subseteq 2^{[k-1]}$ be a full antichain where $\mathcal{J} \neq\left(\begin{array}{c}{[k-1]} \\ k-2\end{array}\right)$. For every $0<\mu, p<1$, there exists $n_{0}$ such that for all $n \geq n_{0}$ with $r \mid n$, there exists an $n$-vertex $k$-graph $H$ which

- satisfies $\operatorname{Disc}^{(k)}\left(\left(\begin{array}{c}{[k]} \\ k-1\end{array}\right), \geq p, \mu\right)$,

- for every $x \in V(H)$ the link $L_{H}(x)$ satisfies $\operatorname{Disc}^{(k-1)}(\mathcal{J}, \geq \alpha, \mu)$,

- there exists $x \in V(H)$ such that the link $L_{H}(x)$ fails $\operatorname{Disc}^{(k-1)}\left(\left(\begin{array}{c}{[k-1]} \\ k-2\end{array}\right), \geq \alpha, \mu\right)$, 
- has no perfect $K_{r}$-packing.

The remainder of this paper is organized as follows. In Sections 2 and 3 we discuss the two main tools needed for the proof of Theorem 1, in Section 4 we prove Theorem 1, and finally in Section 5 we explain the constructions which prove Propositions 3 and 4.

\section{Absorbing Sets}

One of the main tools for our proof of Theorem 1 is the absorbing technique of Rödl-RucińskiSzemerédi [34]. We will use the following absorbing lemma from [28] without modification.

Definition. Let $F$ and $H$ be k-graphs and let $A, B \subseteq V(H)$. We say that $A F$-absorbs $B$ or that $A$ is an $F$-absorbing set for $B$ if both $H[A]$ and $H[A \cup B]$ have perfect $F$-packings. When $F$ is a single edge, we say that $A$ edge-absorbs $B$.

Definition. Let $F$ and $H$ be k-graphs, $\epsilon>0$, and $a$ and $b$ be multiples of $v(F)$. We say that $H$ is $(a, b, \epsilon, F)$-rich if for all $B \in\left(\begin{array}{c}V(H) \\ b\end{array}\right)$ there are at least $\epsilon n^{a}$ sets in $\left(\begin{array}{c}V(H) \\ a\end{array}\right)$ which $F$-absorb $B$.

Lemma 5. (Absorbing Lemma, specialized version of [28, Lemma 10]) Let $F$ be a k-graph, $\epsilon>0$, and $a$ and $b$ be multiples of $v(F)$. There exists an $n_{0}$ and $\omega>0$ such that for all $n$-vertex $k$-graphs $H$ with $n \geq n_{0}$, the following holds. If $H$ is $(a, b, \epsilon, F)$-rich, then there exists an $A \subseteq V(H)$ such that a ||$A \mid$ and $A F$-absorbs all sets $C$ satisfying the following conditions: $C \subseteq V(H)-A,|C| \leq \omega n$, and $b|| C \mid$.

\section{Embedding Lemma}

Definition. Let $k \geq 2$ and $0 \leq m \leq f$. Let $F$ and $H$ be $k$-graphs with $V(F)=\left\{w_{1}, \ldots, w_{f}\right\}$. $A$ labeled copy of $F$ in $H$ is an edge-preserving injection from $V(F)$ to $V(H)$. A degenerate labeled copy of $F$ in $H$ is an edge-preserving map from $V(F)$ to $V(H)$ that is not an injection. Let $1 \leq m \leq f$ and let $Z_{1}, \ldots, Z_{m} \subseteq V(H)$. Set inj $\left[F \rightarrow H ; w_{1} \rightarrow Z_{1}, \ldots, w_{m} \rightarrow Z_{m}\right]$ to be the number of edge-preserving injections $\psi: V(F) \rightarrow V(H)$ such that $\psi\left(w_{i}\right) \in Z_{i}$ for all $1 \leq i \leq m$. If $Z_{i}=\left\{z_{i}\right\}$, we abbreviate $w_{i} \rightarrow\left\{z_{i}\right\}$ as $w_{i} \rightarrow z_{i}$.

The embedding lemma (Lemma 6) proved in this section shows that if $H$ satisfies $\operatorname{Disc}^{(k)}(\mathcal{I}, \geq p, \mu)$ and $\operatorname{Disc}^{(k-1)}(\mathcal{J}, \geq \alpha, \mu)$ in the links, then $H$ contains many copies of $F$ if $F$ is $(\mathcal{I}, \mathcal{J})$-adapted. In fact, it says more: if $m$ of the vertices of $F$ are pre-specified and $F$ satisfies the following more technical condition, then there are many copies of $F$ using the $m$ pre-specified vertices.

Definition. Let $k \geq 2, \mathcal{I} \subseteq 2^{[k]}$ and $\mathcal{J} \subseteq 2^{[k-1]}$ be antichains, $F$ a k-graph, and $s_{1}, \ldots, s_{m} \in$ $V(F)$. We say that $F$ is $(\mathcal{I}, \mathcal{J})$-adapted at $s_{1}, \ldots, s_{m}$ if there exists an ordering $E_{1}, \ldots, E_{t}$ of the edges of $F$ such that 
- for every $i,\left|E_{i} \cap\left\{s_{1}, \ldots, s_{m}\right\}\right| \leq 1$,

- for every $E_{i}$ with $E_{i} \cap\left\{s_{1}, \ldots, s_{m}\right\}=\emptyset$, there exists a bijection $\phi_{i}: E_{i} \rightarrow[k]$ such that for all $j<i$, there exists $I \in \mathcal{I}$ with $\left\{\phi_{i}(x): x \in E_{j} \cap E_{i}\right\} \subseteq I$,

- for every $E_{i}$ with $s_{\ell} \in E_{i}$, there exists a bijection $\psi_{i}: E_{i} \backslash\left\{s_{\ell}\right\} \rightarrow[k-1]$ such that for all $j<i$, there exists $J \in \mathcal{J}$ with $\left\{\psi_{i}(x): x \in E_{j} \cap E_{i}, x \neq s_{\ell}\right\} \subseteq J$.

Note that $m=0$ is possible, in which case the definition is equivalent to $\mathcal{I}$-adapted.

Lemma 6. Let $k \geq 2,0<\alpha, \gamma, p<1$, and $\mathcal{I} \subseteq 2^{[k]}$ and $\mathcal{J} \subseteq 2^{[k-1]}$ be antichains. Let $F$ be an $f$-vertex $k$-graph with $V(F)=\left\{s_{1}, \ldots, s_{m}, t_{m+1}, \ldots, t_{f}\right\}$. Suppose that $F$ is $(\mathcal{I}, \mathcal{J})$ adapted at $s_{1}, \ldots, s_{m}$. Then there exists an $n_{0}$ and $\mu>0$ such that the following is true.

Let $H$ be an $n$-vertex $k$-graph with $n \geq n_{0}$, where $H$ satisfies $\operatorname{Disc}^{(k)}(\mathcal{I}, \geq p, \mu)$. If $m>0$, then also assume that $L_{H}(x)$ satisfies $\operatorname{Disc}^{(k-1)}(\mathcal{J}, \geq \alpha, \mu)$ for every vertex $x \in V(H)$. Let $y_{1}, \ldots, y_{m} \in V(H)$ be distinct and let $V_{m+1}, \ldots, V_{f} \subseteq V(H)$. Then

$$
\begin{aligned}
\operatorname{inj}\left[F \rightarrow H ; s_{1} \rightarrow y_{1}, \ldots,\right. & \left.s_{m} \rightarrow y_{m}, t_{m+1} \rightarrow V_{m+1}, \ldots, t_{f} \rightarrow V_{f}\right] \\
& \geq \alpha^{d_{F}\left(s_{1}\right)} \cdots \alpha^{d_{F}\left(s_{m}\right)} p^{|F|-\sum d_{F}\left(s_{i}\right)}\left|V_{m+1}\right| \cdots\left|V_{f}\right|-\gamma n^{f-m} .
\end{aligned}
$$

Proof. We first prove the lemma under the additional assumption that the sets $V_{m+1}, \ldots, V_{f}$ are pairwise disjoint. This is proved by induction on $|F|$. If $|F|=0$, then

$$
\begin{aligned}
\operatorname{inj}\left[F \rightarrow H ; s_{1} \rightarrow y_{1}, \ldots, s_{m} \rightarrow y_{m}, t_{m+1} \rightarrow V_{m+1}, \ldots, t_{f} \rightarrow V_{f}\right] & \geq \prod_{i=m+1}^{f}\left(\left|V_{i}\right|-f\right) \\
& \geq \alpha^{0} p^{0} \prod_{i=m+1}^{f}\left|V_{i}\right|-\gamma n^{f-m}
\end{aligned}
$$

for large $n$. So assume $F$ has at least one edge and let $E$ be the last edge in an ordering of the edges of $F$ which witness that $F$ is $(\mathcal{I}, \mathcal{J})$-adapted at $s_{1}, \ldots, s_{m}$. (Recall that if $m=0$ then $(\mathcal{I}, \mathcal{J})$-adapted at $s_{1}, \ldots, s_{m}$ is equivalent to $\mathcal{I}$-adapted.)

Let $F_{*}$ be the hypergraph formed by deleting all vertices of $E$ from $F$. Let $F_{-}$be the hypergraph formed by removing the edge $E$ from $F$ but keeping the same vertex set. Let $Q_{*}$ be an injective edge-preserving map $Q_{*}: V\left(F_{*}\right) \rightarrow V(H)$ where $Q_{*}\left(s_{i}\right)=y_{i}$ for $1 \leq i \leq m$ and $Q_{*}\left(t_{j}\right) \in V_{j}$ for $t_{j} \notin E$. There are two cases.

Case 1: $E \cap\left\{s_{1}, \ldots, s_{m}\right\}=\emptyset$. Let $\phi: E \rightarrow[k]$ be the bijection from the definition of $(\mathcal{I}, \mathcal{J})$-adapted at $s_{1}, \ldots, s_{m}$ and assume the vertices of $F$ are labeled such that $E=$ $\left\{t_{m+1}, \ldots, t_{m+k}\right\}$, where $\phi\left(t_{m+i}\right)=i$. For each $I \in \mathcal{I}$, define an $|I|$-uniform hypergraph $\lambda_{I, Q_{*}}$ with vertex set $V(H)$ as follows. Let $I=\left\{i_{1}, \ldots, i_{|I|}\right\}$. Make $\left\{z_{i_{1}}, \ldots, z_{i_{|I|}}\right\} \in\left(\begin{array}{c}V(H) \\ |I|\end{array}\right)$ a hyperedge of $\lambda_{I, Q_{*}}$ if $z_{i_{j}} \in V_{m+i_{j}}$ for all $j$ and when the map $Q_{*}$ is extended to map $t_{i_{j}}$ to $z_{i_{j}}$ for all $j$, this extended map is an edge-preserving map from $F_{-}\left[V\left(F_{*}\right) \cup\left\{t_{i_{1}}, \ldots, t_{i_{|I|}}\right\}\right]$ to $H$. More informally, $\lambda_{I, Q_{*}}$ consists of all $|I|$-sets to which $Q_{*}$ can be extended to produce a copy of $F_{*}$ together with the vertices of $E$ indexed by $I$. Let $\Lambda_{Q_{*}}=\left(\lambda_{I, Q_{*}}\right)_{I \in \mathcal{I}}$. 
Now, if $\left(z_{m+1}, \ldots, z_{m+k}\right)$ is a $k$-tuple in $K_{k}\left(\Lambda_{Q_{*}}\right)$, then the map $Q_{*}$ can be extended to map $t_{j}$ to $z_{j}$ for $m+1 \leq j \leq m+k$ to produce an edge-preserving map from $F_{-}$to $H$. To see this, let $E^{\prime}$ be an edge of $F_{-}$. Since $E$ is the last edge in the ordering, if $E^{\prime} \cap E=\left\{t_{j_{1}}, \ldots, t_{j_{r}}\right\}$ then there exists some $I \in \mathcal{I}$ with $\left\{j_{1}, \ldots, j_{r}\right\} \subseteq I$ since $F$ is $\mathcal{I}$-adapted. Since $\left(z_{m+1}, \ldots, z_{m+k}\right)$ is a $k$-clique, $\left\{z_{m+i}: i \in I\right\} \in \lambda_{I, Q_{*}}$. This implies that there is some permutation $\eta$ of $I$ such that extending $Q_{*}$ to map $t_{m+i}$ to $z_{m+\eta(i)}$ produces an edge-preserving map. Since the $V_{m+i} \mathrm{~S}$ are pairwise disjoint and $z_{m+i} \in V_{m+i}$ for all $i \in I, \eta$ must be the identity permutation, i.e. extending the map $Q_{*}$ to map $t_{m+i}$ to $z_{m+i}$ for all $i \in I$ produces an edge-preserving map. Thus extending the map $Q_{*}$ to map $t_{j_{p}}$ to $z_{j_{p}}$ for all $p$ is an edge-preserving map and $E^{\prime}$ is one of the preserved edges. Finally, since the $V_{j}$ s are disjoint, each $k$-tuple in $K_{k}\left(\Lambda_{Q_{*}}\right)$ corresponds to exactly one labeled copy of $F_{-}$in $H$ which extend $Q_{*}$ with $t_{j}$ mapped into $V_{j}$ for all $j$. Similarly, $\left|H \cap K_{k}\left(\Lambda_{Q_{*}}\right)\right|$ is exactly the number of labeled copies of $F$ in $H$ which extend $Q_{*}$ with $t_{j}$ mapped into $V_{j}$ for all $j$. Thus,

$$
\begin{aligned}
\operatorname{inj}[F & \left.\rightarrow H ; s_{1} \rightarrow y_{1}, \ldots, s_{m} \rightarrow y_{m}, t_{m+1} \rightarrow V_{m+1}, \ldots, t_{f} \rightarrow V_{f}\right]=\sum_{Q_{*}}\left|H \cap K_{k}\left(\Lambda_{Q_{*}}\right)\right| \\
\operatorname{inj}\left[F_{-} \rightarrow H ; s_{1} \rightarrow y_{1}, \ldots, s_{m} \rightarrow y_{m}, t_{m+1}\right. & \left.\rightarrow V_{m+1}, \ldots, t_{f} \rightarrow V_{f}\right]=\sum_{Q_{*}}\left|K_{k}\left(\Lambda_{Q_{*}}\right)\right| .
\end{aligned}
$$

Since $H$ satisfies $\operatorname{Disc}^{(k)}(\mathcal{I}, \geq p, \mu)$,

$$
\begin{array}{r}
\operatorname{inj}\left[F \rightarrow H ; s_{1} \rightarrow y_{1}, \ldots, s_{m} \rightarrow y_{m}, t_{m+1} \rightarrow V_{m+1}, \ldots, t_{f} \rightarrow V_{f}\right] \\
\geq \sum_{Q_{*}}\left(p\left|K_{k}\left(\Lambda_{Q_{*}}\right)\right|-\mu n^{k}\right) \\
\geq p \sum_{Q_{*}}\left|K_{k}\left(\Lambda_{Q_{*}}\right)\right|-\mu n^{f-m},
\end{array}
$$

where the last inequality is because there are at most $n^{f-m-k}$ maps $Q_{*}$, since $F_{*}$ has $f-k$ vertices and $s_{i} \in V\left(F_{*}\right)$ must map to $y_{i}$. Combining (1) and (2) and then applying induction,

$$
\begin{aligned}
\operatorname{inj}[F \rightarrow H ; & \left.s_{1} \rightarrow y_{1}, \ldots, s_{m} \rightarrow y_{m}, t_{m+1} \rightarrow V_{m+1}, \ldots, t_{f} \rightarrow V_{f}\right] \\
& \geq p \operatorname{inj}\left[F_{-} \rightarrow H ; s_{1} \rightarrow y_{1}, \ldots, s_{m} \rightarrow y_{m}, t_{m+1} \rightarrow V_{m+1}, \ldots, t_{f} \rightarrow V_{f}\right]-\mu n^{f-m} \\
& \geq p\left(\alpha^{\sum d\left(s_{i}\right)} p^{|F|-1-\sum d\left(s_{i}\right)}\left|V_{m+1}\right| \cdots\left|V_{f}\right|-\gamma n^{f-m}\right)-\mu n^{f-m} .
\end{aligned}
$$

Let $\mu=(1-p) \gamma$ so that the proof of this case complete.

Case 2: $s_{\ell} \in E$. (Since $F$ is $(\mathcal{I}, \mathcal{J})$-adapted at $s_{1}, \ldots, s_{m}$, at most one vertex $s_{\ell}$ can be in $E$.) Let $\psi: E \backslash\left\{s_{\ell}\right\} \rightarrow[k-1]$ be the bijection from the definition of $(\mathcal{I}, \mathcal{J})$-adapted at $s_{1}, \ldots, s_{m}$ and assume the vertices of $E$ are labeled such that $E=\left\{s_{\ell}, t_{m+1}, \ldots, t_{m+k-1}\right\}$ where $\psi\left(t_{m+j}\right)=j$. This case is very similar to the previous case, except we will use $\operatorname{Disc}^{(k-1)}(\mathcal{J}, \geq \alpha, \mu)$ in the link of $y_{\ell}$. For each $J \in \mathcal{J}$, define a $|J|$-uniform hypergraph $\lambda_{J, Q_{*}}$ with vertex set $V(H)$ as follows. Let $J=\left\{j_{1}, \ldots, j_{|J|}\right\}$. Make $\left\{z_{j_{1}}, \ldots, z_{j_{|J|}}\right\}$ a hyperedge of $\lambda_{J, Q_{*}}$ if $z_{j_{r}} \in V_{j_{r}}$ for all $r$ and extending the map $Q_{*}$ to map $s_{\ell}$ to $y_{\ell}$ and mapping $t_{j_{r}}$ 
to $z_{j_{r}}$ for all $r$ produces an edge-preserving map. Let $\Lambda_{Q_{*}}=\left(\lambda_{J, Q_{*}}\right)_{J \in \mathcal{J}}$. Similar to before, if $\left(z_{m+1}, \ldots, z_{m+k-1}\right)$ is a $(k-1)$-tuple in $K_{k-1}\left(\Lambda_{Q_{*}}\right)$, then the map $Q_{*}$ can be extended to map $s_{\ell}$ to $y_{\ell}$ and map $t_{i}$ to $z_{i}$ for $m+1 \leq i \leq m+k-1$ to produce an edge-preserving map from $F_{-}$to $H$. Thus $\left|K_{k-1}\left(\Lambda_{Q_{*}}\right)\right|$ is exactly the number of labeled copies of $F_{-}$in $H$ which extend $Q_{*}$. Similarly, $\left|L_{H}\left(y_{\ell}\right) \cap K_{k-1}\left(\Lambda_{Q_{*}}\right)\right|$ is exactly the number of labeled copies of $F$ in $H$ which extend $Q_{*}$.

Now formulas similar to (11) and (2) and the fact that $L_{H}\left(y_{\ell}\right)$ satisfies $\operatorname{Disc}^{(k-1)}(\mathcal{J}, \geq \alpha, \mu)$ completes this case. This concludes the proof of the lemma if the sets $V_{m+1}, \ldots, V_{f}$ are pairwise disjoint.

Now assume that the sets $V_{m+1}, \ldots, V_{f}$ are not necessarily pairwise disjoint. Let $\mathcal{P}=$ $\left\{\left(P_{m+1}, \ldots, P_{f}\right): P_{m+1}, \ldots, P_{f}\right.$ is a partition of $\left.V(H)\right\}$ so that $|\mathcal{P}|=(f-m)^{n}$. Now

$$
\begin{aligned}
& \operatorname{inj}\left[F \rightarrow H ; s_{1} \rightarrow y_{1}, \ldots, s_{m} \rightarrow y_{m}, t_{m+1} \rightarrow V_{m+1}, \ldots, t_{f} \rightarrow V_{f}\right] \frac{1}{(f-m)^{n-f+m}} \sum_{\left(P_{m+1}, \ldots, P_{f}\right) \in \mathcal{P}} \operatorname{inj}\left[F \rightarrow H ; s_{1} \rightarrow y_{1}, \ldots, s_{m} \rightarrow y_{m},\right. \\
&\left.t_{m+1} \rightarrow V_{m+1} \cap P_{m+1}, \ldots, t_{f} \rightarrow V_{f} \cap P_{f}\right] .
\end{aligned}
$$

Indeed, each labeled copy of $F$ of the right form will be counted exactly $(f-m)^{n-f+m}$ times by the sum over all partitions, since the images of $t_{m+1}, \ldots, t_{f}$ must map into the cooresponding part of the partition and all other vertices of $H$ can be distributed to any of the parts of the partition. Let $\delta=\alpha^{d_{F}\left(s_{1}\right)} \cdots \alpha^{d_{F}\left(s_{m}\right)} p^{|F|-\sum d_{F}\left(s_{i}\right)}$. Since $V_{m+1} \cap P_{m+1}, \ldots, V_{f} \cap P_{f}$ are pairwise disjoint,

$$
\begin{aligned}
\operatorname{inj}\left[F \rightarrow H ; s_{1}\right. & \left.\rightarrow y_{1}, \ldots, s_{m} \rightarrow y_{m}, t_{m+1} \rightarrow V_{m+1}, \ldots, t_{f} \rightarrow V_{f}\right] \\
\geq & \frac{1}{(f-m)^{n-f+m}} \sum_{\left(P_{m+1}, \ldots, P_{f}\right) \in \mathcal{P}}\left(\delta\left|V_{m+1} \cap P_{m+1}\right| \cdots\left|V_{f} \cap P_{f}\right|-\gamma n^{f-m}\right) \\
= & \delta\left|V_{m+1}\right| \cdots\left|V_{f}\right|-\frac{\gamma n^{f-m}|\mathcal{P}|}{(f-m)^{n-f+m}} \geq \delta\left|V_{m+1}\right| \cdots\left|V_{f}\right|-\gamma n^{f-m} .
\end{aligned}
$$

\section{Packing $(\mathcal{I}, \mathcal{J})$-adapted hypergraphs}

In this section we prove Theorem 1. The proof has several stages: we first prove that the quasirandom conditions on $H$ imply that $H$ is rich, then we use Lemma 5 to set aside a vertex set $A$ which can absorb all reasonably sized sets, next we use the embedding lemma (Lemma 6) to produce an almost perfect packing in $H-A$, and finally we use the properties of $A$ to absorb the remaining vertices.

\subsection{Richness}

In this subsection, we prove that the conditions on $H$ in Theorem 1 imply that $H$ is $\left(f^{2}-\right.$ $f, f, \epsilon, F)$-rich, where $f=v(F)$. 
Lemma 7. Let $k \geq 2, \mathcal{I} \subseteq 2^{[k]}$ be a full antichain, and $\mathcal{J} \subseteq 2^{[k-1]}$ an antichain. Let $F$ be an $(\mathcal{I}, \mathcal{J})$-adapted $k$-graph with $f$ vertices. For every $0<\alpha, p<1$, there exists $\mu, \epsilon>0$ and $n_{0}$ so that the following holds. Let $H$ be an $n$-vertex $k$-graph where $n \geq n_{0}$. Also, assume that $H$ satisfies Disc ${ }^{(k)}(\mathcal{I}, \geq p, \mu)$ and that $L_{H}(z)$ satisfies $\operatorname{Disc}^{(k-1)}(\mathcal{J}, \geq \alpha, \mu)$ for every vertex $z \in V(H)$. Then $H$ is $\left(f^{2}-f, f, \epsilon, F\right)$-rich.

Proof. Let $a=f(f-1)$ and $b=f$. Our task is to come up with an $\epsilon>0$ such that for large $n$ and all $B \in\left(\begin{array}{c}V(H) \\ b\end{array}\right)$, there are at least $\epsilon n^{a}$ vertex sets of size $a$ which $F$-absorb $B$; we will define $\epsilon$ and $\mu$ later. Let $V(F)=\left\{w_{0}, \ldots, w_{f-1}\right\}$, where $w_{0}$ is the special vertex in the definition that $F$ is $(\mathcal{I}, \mathcal{J})$-adapted.

Next, form the following $k$-graph $F^{\prime}$. Let

$$
V\left(F^{\prime}\right)=\left\{x_{i, j}: 0 \leq i, j \leq f-1\right\} .
$$

(We think of the vertices of $F^{\prime}$ as arranged in a grid with $i$ as the row and $j$ as the column.) Form the edges of $F^{\prime}$ as follows: for each fixed $1 \leq i \leq f-1$, let $\left\{x_{i, 0}, \ldots, x_{i, f-1}\right\}$ induce a copy of $F$ where $x_{i, j}$ is mapped to $w_{j}$. Similarly, for each fixed $0 \leq j \leq f-1$, let $\left\{x_{0, j}, \ldots, x_{f-1, j}\right\}$ induce a copy of $F$ where $x_{i, j}$ is mapped to $w_{i}$. Note that we therefore have a copy of $F$ in each column and a copy of $F$ in each row besides the zeroth row.

Now fix $B=\left\{b_{0}, \ldots, b_{f-1}\right\} \subseteq V(H)$; we want to show that $B$ is $F$-absorbed by many $a$-sets. Note that any labeled copy of $F^{\prime}$ in $H$ which maps $x_{0,0} \rightarrow b_{0}, \ldots, x_{0, f-1} \rightarrow b_{f-1}$ produces an $F$-absorbing set for $B$ as follows. Let $Q: V\left(F^{\prime}\right) \rightarrow V(H)$ be an edge-preserving injection where $Q\left(b_{j}\right)=x_{0, j}$ (so $Q$ is a labeled copy of $F^{\prime}$ in $H$ where the set $B$ is the zeroth row of $\left.F^{\prime}\right)$. Let $A=\left\{Q\left(x_{i, j}\right): 1 \leq i \leq f-1,0 \leq j \leq f-1\right\}$ consist of all vertices in rows 1 through $f-1$. Then $A$ has a perfect $F$-packing consisting of the copies of $F$ on the rows, and $A \cup B$ has a perfect $F$-packing consisting of the copies of $F$ on the columns. Therefore, $A F$-absorbs $B$.

To complete the proof, we therefore just need to use Lemma 6 where $m=f$ and $s_{1}=$ $x_{0,0}, \ldots, s_{f}=x_{0, f-1}$ to show that there are many copies of $F^{\prime}$ with $B$ as the zeroth row. To do so, we need to show that $F^{\prime}$ is $(\mathcal{I}, \mathcal{J})$-adapted at $s_{1}, \ldots, s_{m}$. Indeed, consider the following ordering of edges of $F^{\prime}$. First, list the edges of $F^{\prime}$ in the first column, then the edges of $F^{\prime}$ in the second column, and so on until the $k$ th column. Next, list the edges of $F^{\prime}$ in the first row, then the second row, and so on until the $(k-1)$ st row. Within each row or column, list the edges in the ordering given in the definition of $F$ being $(\mathcal{I}, \mathcal{J})$-adapted. For the bijections $\phi$ or $\psi$, use the same bijection as in the definition of $F$ being $(\mathcal{I}, \mathcal{J})$-adapted. Now consider $E_{i}, E_{j} \in F^{\prime}$ in this ordering with $j<i$. If $E_{i}$ and $E_{j}$ are from the same row or the same column, then since $F$ is $(\mathcal{I}, \mathcal{J})$-adapted the condition on $E_{i} \cap E_{j}$ is satisfied. If $E_{i}$ and $E_{j}$ are in different rows or columns, the size of their intersection is at most one. If $E_{i} \cap E_{j}=\emptyset$ then the condition is trivially satisfied. If $E_{i} \cap E_{j}=\{u\}$, then $E_{i}$ must be from a row since $i>j$. Then $E_{i}$ does not contain any $s_{1}, \ldots, s_{m}$, so we must show that there is some $I \in \mathcal{I}$ so that $\phi_{i}(u) \in I$. This is true because $\mathcal{I}$ is full. Thus $F^{\prime}$ is $(\mathcal{I}, \mathcal{J})$-adapted at $s_{1}, \ldots, s_{m}$.

Now apply Lemma 6 to $F^{\prime}$ with $m=f, s_{1}=x_{0,0}, \ldots, s_{f}=x_{0, f-1}, V_{m+1}=\cdots=V_{f^{2}}=$ $V(H)-B$, and $\gamma=\frac{1}{2} \alpha^{\sum d\left(x_{0, j}\right)} p^{|F|-\sum d\left(x_{0, j}\right)}$. Ensure that $n_{0}$ is large enough and $\mu$ is small 
enough apply Lemma 6 to show that

$$
\operatorname{inj}\left[F^{\prime} \rightarrow H ; x_{0,0} \rightarrow b_{0}, \ldots, x_{0, f-1} \rightarrow b_{f-1}\right] \geq \gamma\left(\frac{n}{2}\right)^{f^{2}-f}=\frac{\gamma}{2^{f^{2}-f}} n^{a} .
$$

Each labeled copy of $F^{\prime}$ produces a labeled $F$-absorbing set for $B$, so there are at least $\frac{\gamma}{a ! 2^{f^{2}-f}} n^{a} F$-absorbing sets for $B$. The proof is complete by letting $\epsilon=\frac{\gamma}{a ! 2^{f^{2}-f}}$.

\subsection{Almost perfect packings}

In this section we prove that the conditions in Theorem 1 imply that there exists a perfect $F$-packing covering almost all the vertices of $H$.

Lemma 8. Let $k \geq 2$ and $\mathcal{I} \subseteq 2^{[k]}$ be a full antichain. Fix $0<p<1$ and an $\mathcal{I}$-adapted $k$-graph $F$ with $f$ vertices. Fix an integer $b$ with $f \mid b$. For any $0<\omega<1$, there exists $n_{0}$ and $\mu>0$ such that the following holds. Let $H$ be an $k$-graph satisfying $\operatorname{Disc}^{(k)}(\mathcal{I}, \geq p, \mu)$ with $n \geq n_{0}$ and $f \mid n$. Then there exists $C \subseteq V(H)$ such that $|C| \leq \omega n, b|| C \mid$, and $H[\bar{C}]$ has a perfect F-packing.

Proof. First, select $n_{0}$ large enough and $\mu$ small enough so that any vertex set $C$ of size $\left\lceil\frac{\omega}{2}\right\rceil$ contains a copy of $F$. To see this, let $\gamma=\frac{1}{2} p^{|F|}\left(\frac{\omega}{2}\right)^{f}$ and select $n_{0}$ and $\mu>0$ according to Lemma 6 with $m=0$. (Recall that if $m=0$ then the condition $(\mathcal{I}, \mathcal{J})$-adapted on $F$ at $\emptyset$ just reduces to the statement that $F$ is $\mathcal{I}$-adapted.) Now if $C \subseteq V(H)$ with $|C| \geq \frac{\omega}{2} n$, then let $V_{1}=\cdots=V_{f}=C$ so that $\left|V_{i}\right| \geq \frac{\omega}{2}$ for all $i$. Then Lemma 6 implies there are at least $p^{|F|} \prod\left|V_{i}\right|-\gamma n^{f} \geq p^{|F|}\left(\frac{\omega}{2}\right)^{f} n^{f}-\gamma n^{f}=\gamma n^{f}>0$ copies of $F$ inside $C$.

Now let $F_{1}, \ldots, F_{t}$ be a greedily constructed $F$-packing. That is, $F_{1}, \ldots, F_{t}$ are disjoint copies of $F$ and $C:=V(H)-V\left(F_{1}\right)-\cdots-V\left(F_{t}\right)$ has no copy of $F$. By the previous paragraph, $|C| \leq \frac{\omega}{2} n$. Since $f \mid n$ and $H[\bar{C}]$ has a perfect $F$-packing, $f|| C \mid$. Thus we can let

$y \equiv-\frac{|C|}{f}(\bmod b)$ with $0 \leq y<b$ and take $y$ of the copies of $F$ in the $F$-packing of $H[\bar{C}]$ and add their vertices into $C$ so that $b|| C \mid$.

\subsection{Proof of Theorem 1}

Proof of Theorem 1. First, apply Lemma 7 to produce $\epsilon>0$. Next, select $\omega>0$ according to Lemma 5 and $\mu_{1}>0$ according to Lemma 8. Also, make $n_{0}$ large enough so that both Lemma 5 and 8 can be applied. Let $\mu=\mu_{1} \omega^{k}$. All the parameters have now been chosen.

By Lemmas 5 and 7, there exists a set $A \subseteq V(H)$ such that $A F$-absorbs $C$ for all $C \subseteq V(H) \backslash A$ with $|C| \leq \omega n$ and $b|| C \mid$. If $|A| \geq(1-\omega) n$, then $A F$-absorbs $V(H) \backslash A$ so that $H$ has a perfect $F$-packing. Thus $|A| \leq(1-\omega) n$. Next, let $H^{\prime}:=H[\bar{A}]$ and notice that $H^{\prime}$ satisfies $\operatorname{Disc}^{(k)}\left(\mathcal{I}, \geq p, \mu_{1}\right)$ since $v\left(H^{\prime}\right) \geq \omega n$ and

$$
\mu n^{k} \leq \frac{\mu}{\omega^{k}} v\left(H^{\prime}\right)^{k}=\mu_{1} v\left(H^{\prime}\right)^{k} .
$$

Therefore, by Lemma 8, there exists a vertex set $C \subseteq V\left(H^{\prime}\right)=V(H) \backslash A$ such that $|C| \leq \omega n$, $|C|$ is a multiple of $b$, and $H^{\prime}[\bar{C}]$ has a perfect $F$-packing. Now Lemma 5 implies that $A$ 
$F$-absorbs $C$. The perfect $F$-packing of $A \cup C$ and the perfect $F$-packing of $H^{\prime}[\bar{C}]$ produces a perfect $F$-packing of $H$.

\section{Constructions}

In this section, we prove Propositions 3 and 4 using the following construction.

Construction. Let $k \geq 2$. Let $A_{n}^{(k)}$ be the following probability distribution over $n$-vertex $k$-graphs. Let $f:\left(\begin{array}{c}V\left(A_{n}^{(k)}\right) \\ k-1\end{array}\right) \rightarrow\{0, \ldots, k-1\}$ be a random $k$-coloring of the $(k-1)$-sets. Make $E \in\left(\begin{array}{c}V\left(A_{n}^{(k)}\right) \\ k\end{array}\right)$ an edge of $A_{n}^{(k)}$ if

$$
\sum_{\substack{T \subseteq E \\|T|=k-1}} f(T) \neq 0 \quad(\bmod k) .
$$

Lemma 9. Let $p=\frac{k-1}{k}$ and $\epsilon>0$. Then with probability going to one as $n$ goes to infinity,

$$
|| A_{n}^{(k)}\left|-p\left(\begin{array}{l}
n \\
k
\end{array}\right)\right|<\epsilon n^{k} .
$$

Proof. Each $k$-set is an edge with probability exactly $p$, so $\mathbb{E}\left[\left|A_{n}^{(k)}\right|\right]=p\left(\begin{array}{l}n \\ k\end{array}\right)$. A simple second moment argument then shows that with high probability the number of edges is concentrated around $p\left(\begin{array}{l}n \\ k\end{array}\right)$.

Lemma 10. There exists a $\mu_{0}$ such that for all $0<\mu<\mu_{0}$, with probability going to one as $n$ goes to infinity, $A_{n}^{(k)}$ fails Disc ${ }^{(k)}\left(\left(\begin{array}{c}{[k]} \\ k-1\end{array}\right), \geq p, \mu\right)$.

Proof. Let $Z$ be the $(k-1)$-graph whose edges are all the $(k-1)$-sets colored zero. Let $\Lambda=(Z, \ldots, Z)$ be the $\left(\begin{array}{c}{[k]} \\ k-1\end{array}\right)$-layout consisting of $Z$ in every coordinate. Now any $k$-clique $\left(z_{1}, \ldots, z_{k}\right)$ of $\Lambda$ is not a hyperedge of $A_{n}^{(k)}$, since every $(k-1)$-subset of $\left\{z_{1}, \ldots, z_{k}\right\}$ has color zero. This $\Lambda$ will show that $A_{n}^{(k)}$ fails $\operatorname{Disc}^{(k)}\left(\left(\begin{array}{c}{[k]} \\ k-1\end{array}\right), \geq p, \mu\right)$ if $\left|K_{k}(\Lambda)\right|$ is large enough. Each $k$-tuple of vertices is a $k$-clique with probability $\left(\frac{1}{k}\right)^{k}$, so $\mathbb{E}\left[\left|K_{k}(\Lambda)\right|\right]=k^{-k}(n)_{k}$. A simple second moment computation shows that $\left|K_{k}(\Lambda)\right|$ is concentrated around its expectation, so with high probability for large $n$ we have that $\left|K_{k}(\Lambda)\right| \geq \frac{1}{10} k^{-k} n^{k}$. Thus if $\mu_{0}=\frac{1}{20} \frac{k-1}{k^{k+1}}$, we have that

$$
0=\left|H \cap K_{k}(\Lambda)\right|<\frac{k-1}{k}\left|K_{k}(\Lambda)\right|-\mu n^{k}
$$

Lemma 11. Let $r=r_{k-1}\left(K_{k}^{(k-1)}, \ldots, K_{k}^{(k-1)}\right)$ be the $k$-color Ramsey number, where the $(k-1)$-sets are colored and a monochromatic $k$-clique is forced. Then $A_{n}^{(k)}$ has no copy of $K_{r}^{(k)}$. 
Proof. Let $X \subseteq V\left(A_{n}^{(k)}\right)$ be such that $|X|=r$ and $A_{n}^{(k)}[X]$ is a clique. Then by the property of $r$, there exists a $Y \subseteq X$ such that $|Y|=k$ and all $(k-1)$-subsets of $Y$ have the same color $c$. But now

$$
\sum_{\substack{T \subseteq Y \\|T|=k-1}} f(T)=c k=0 \quad(\bmod k) .
$$

Thus $Y \notin A_{n}^{(k)}$, which contradicts that $A_{n}^{(k)}[X]$ is a clique.

To show that $A_{n}^{(k)}$ satisfies $\operatorname{Disc}^{(k)}(\mathcal{I}, \geq p, \mu)$ when $\mathcal{I} \neq\left(\begin{array}{c}{[k]} \\ k-1\end{array}\right)$, we will use a theorem of Towsner [38] that equates $\mathcal{I}$-discrepency with counting $\mathcal{I}$-adapted hypergraphs. Therefore, we prove that the count of any $\mathcal{I}$-adapted hypergraph $F$ in $A_{n}^{(k)}$ is correct with high probability.

Lemma 12. Let $p=\frac{k-1}{k}$ and let $\mathcal{I} \subseteq 2^{[k]}$ be an antichain such that $\mathcal{I} \neq\left(\begin{array}{c}\left.{ }_{k-1}{ }_{k}\right] \\ )\end{array}\right)$. Let $F$ be an $\mathcal{I}$-adapted $k$-graph. For every $\mu>0$, with probability going to one as $n$ goes to infinity, the number of labeled copies of $F$ in $A_{n}^{(k)}$ satisfies

$$
\left|\operatorname{inj}\left[F \rightarrow A_{n}^{(k)}\right]-p^{|F|} n^{v(F)}\right|<\mu n^{v(F)} .
$$

Proof. Let $E_{1}, \ldots, E_{m}$ be the ordering of edges in the definition of $F$ being $\mathcal{I}$-adapted. First we shows that if $Q: V(F) \rightarrow V\left(A_{n}^{(k)}\right)$ is any injection, then the probability that $Q\left(E_{i}\right) \in A_{n}^{(k)}$ is exactly $p$ independently of if the edges $E_{j}$ with $j<i$ map to hyperedges or not. Indeed, since $\mathcal{I} \neq\left(\begin{array}{c}{[k]} \\ k-1\end{array}\right)$, let $I \in\left(\begin{array}{c}{[k]} \\ k-1\end{array}\right)-\mathcal{I}$. Now consider some $E_{i}$ and let $\phi_{i}: E_{i} \rightarrow[k]$ be the bijection from the definition of $F$ being $\mathcal{I}$-adapted. Now since $I \notin \mathcal{I}$, there is no $j<i$ such that $\phi_{i}\left(E_{i} \cap E_{j}\right)=I$. Thus conditioning on if the edges $E_{j}$ with $j<i$ map to edges of $A_{n}^{(k)}$ or not potentially fixes the colors on $(k-1)$-subsets of $Q\left(E_{i}\right)$ besides the $(k-1)$-subset indexed by $I$. Since the color of $\left\{Q(x): x \in E_{i}, \phi_{i}(x) \in I\right\}$ (which has size $k-1$ ) has probability exactly $p$ to make the color sum of $Q\left(E_{i}\right)$ once all other colors are fixed, with probability $p$ we have that $Q\left(E_{i}\right)$ is an edge.

Therefore, the probability that $Q$ is an edge-preserving map is $p^{|F|}$. This implies that the expected number of labeled copies of $F$ in $A_{n}^{(k)}$ is $p^{|F|} n(n-1) \cdots(n-v(F)+1)$. A simple second moment calculation shows that with high probability the number of labeled copies of $F$ in $A_{n}^{(k)}$ is $p^{|F|} n^{v(F)} \pm \mu n^{v(F)}$ for large $n$.

Lastly, we need to show that $A_{n}^{(k)}$ satisfies $\operatorname{Disc}^{(k-1)}(\mathcal{J}, \geq \alpha, \mu)$ in every link for every $\mathcal{J}$. We could do that similar to the previous lemma by showing that the count of $\mathcal{J}$-adapted $k$-graphs is correct, but instead are able to directly show that $\operatorname{Disc}^{(k-1)}(\mathcal{J}, \geq \alpha, \mu)$ holds.

Lemma 13. Let $\mathcal{J} \subseteq 2^{[k-1]}$ be an antichain and $\alpha=\frac{k-1}{k}$. Then for every $\mu>0$, with probability going to one as $n$ goes to infinity, $L(x)$ satisfies $\operatorname{Disc}^{(k-1)}(\mathcal{J}, \geq \alpha, \mu)$ for each $x \in V\left(A_{n}^{(k)}\right)$. 
Proof. Fix $x \in V\left(A_{n}^{(k)}\right)$ and view $L_{A_{n}^{(k)}}(x)$ as a probability distribution over $(k-1)$-graphs with vertex set $V\left(A_{n}^{(k)}\right)-x$. That is, an element from this probability distribution is generated by first generating $A_{n}^{(k)}$ and then outputting the link of $x$. We claim that the probability distribution $L(x)$ is isomorphic to the probability distribution $G^{(k-1)}(n-1, \alpha)$. To see this, consider $S \in\left(\begin{array}{c}V\left(A_{n}^{(k)}\right)-x \\ k-1\end{array}\right)$. Then $S \in L(x)$ if

$$
\sum_{\substack{T \subseteq S \cup\{x\} \\|T|=k-1}} f(T) \neq 0 \quad(\bmod k) .
$$

We could rewrite this as

$$
f(S) \neq \sum_{\substack{T \subseteq S \\|T|=k-2}} f(T \cup x) \quad(\bmod k) .
$$

The sum on the left hand side is some integer $w_{S}$ between 0 and $k-1$, so that $S$ is a hyperedge of $L(x)$ if and only if the color of $S$ is not $w_{S}$. Since this is for every $S$ and the colors assigned to $S$ are mutually independent, $L(x)$ is isomorphic to $G^{(k-1)}(n-1, \alpha)$.

The proof is now complete, since for large $n G^{(k-1)}(n-1, \alpha)$ satisfies $\operatorname{Disc}^{(k-1)}(\mathcal{J}, \geq \alpha, \mu)$ with very high probability as follows. Fix any $\mathcal{J}$-layout $\Lambda$. Each $(k-1)$-clique in $\Lambda$ is a hyperedge with probability $\alpha$ and two $(k-1)$-cliques are independent unless one is a permutation of the other. So divide $K_{k-1}(\Lambda)$ up into at most $(k-1)$ ! sets $R_{1}, \ldots, R_{(k-1)}$ ! such that within a single $R_{i}$ there are no $(k-1)$-tuples which are permutations of each other. Then the expected size of $H \cap R_{i}$ is $\alpha\left|R_{i}\right|$ and by Chernoff's inequality,

$$
\mathbb{P}\left[|| H \cap R_{i}|-\alpha| R_{i}||>\epsilon n^{k-1}\right]<2 e^{-\epsilon^{2} n^{2 k-2} / 2\left|R_{i}\right|} .
$$

Since $\left|R_{i}\right| \leq n^{k-1}$, the probability is at most $e^{-c n^{k-1}}$ for some constant $c$. There are $(k-1)$ ! sets $R_{i}$ and there are at most $2^{k-2} 2^{n^{k-2}} \mathcal{J}$-layouts $\Lambda$, so with probability at most $e^{-c^{\prime} n^{k-1}}$, the link of $x$ fails $\operatorname{Disc}^{(k-1)}(\mathcal{J}, \geq \alpha, \mu)$. There are $n$ vertices of $A_{n}^{(k)}$, so with probability at most $n e^{-c^{\prime} n^{k-1}} \rightarrow 0$, there is some vertex $x$ of $A_{n}^{(k)}$ whose link fails $\operatorname{Disc}^{(k-1)}(\mathcal{J}, \geq \alpha, \mu)$.

Proof of Proposition 3. As mentioned previously, to show that $A_{n}^{(k)} \operatorname{satisfies~} \operatorname{Disc}^{(k)}(\mathcal{I}, \geq p, \mu)$, we combine Lemma 12 with a theorem of Towsner [38] which is stated in the language of $k$-graph sequences. Converting from the probability distribution $A_{n}^{(k)}$ to a $k$-graph sequence is very similar to the proofs of [29, Lemmas 30 and 31] so we only briefly sketch the technique here. By the previous lemmas and the probabilistic method, for every $\mu>0$ there exists an $n_{0}$ such that for every $n \geq n_{0}$ there exists some $k$-graph satisfying the properties in the previous lemmas (has the right edge density, fails $\operatorname{Disc}{ }^{(k)}\left(\left(\begin{array}{c}{[k]} \\ k-1\end{array}\right), \geq p, \mu\right)$, no copy of $K_{r}$, has the right count of all $\mathcal{I}$-adapted hypergraphs, and satisfies $\operatorname{Disc}^{(k-1)}(\mathcal{J}, \geq \alpha, \mu)$ in the links). Construct a $k$-graph sequence $\mathcal{H}=\left\{H_{n}\right\}_{n \in \mathbb{N}}$ by diagonalization by setting $\mu=\frac{1}{n}$.

By Lemma 12, $\mathcal{H}$ satisfies the property that for every $\mathcal{I}$-adapted $F, \lim _{n \rightarrow \infty} t_{F}\left(H_{n}\right)=p^{|F|}$ so by [38, Theorem 1.1] $\mathcal{H}$ is $\operatorname{Disc}_{p}[\mathcal{I}]\left(\right.$ where $t_{F}\left(H_{n}\right)$ and $\operatorname{Disc}_{p}[\mathcal{I}]$ are defined in [38]). Thus for large $n$, the $k$-graphs in the sequence $\mathcal{H}$ are the $k$-graphs which prove Proposition 3 . 
Proof of Proposition 4. Let $G=G^{(k)}(n, p)$ be the random $k$-graph with density $p$. Modify $G$ by picking a single vertex $x \in V(G)$, removing all edges which contain $x$, and adding edges so that $L(x)=A_{n}^{(k-1)}$. Now the link of $x$ has no copy of $K_{r}^{(k-1)}$ so that $G$ has no perfect $K_{r+1}^{(k)}$-packing. Also, $G$ satisfies $\operatorname{Disc}^{(k)}\left(\left(\begin{array}{c}{[k]} \\ k-1\end{array}\right), \geq p, \mu\right)$ since the random $k$-graph satisfies $\operatorname{Disc}^{(k)}\left(\left(\begin{array}{c}{[k]} \\ k-1\end{array}\right), \geq p, \mu\right)$ (see the proof of Lemma 13) and we only modified at most $n^{k-1}$ hyperedges. By the previous lemmas, the link of $x$ fails $\operatorname{Disc}^{(k-1)}\left(\left(\begin{array}{c}{[k-1]} \\ k-2\end{array}\right), \geq \alpha, \mu\right)$ and satisfies $\operatorname{Disc}^{(k-1)}(\mathcal{J}, \geq \alpha, \mu)$ for all $\mathcal{J} \neq\left(\begin{array}{c}k-1] \\ k-2\end{array}\right)$.

Acknowledgments: The authors would like to thank Daniela Kühn for suggesting the relationship of this work to the Hypergraph Blowup Lemma.

\section{References}

[1] N. Alon and R. Yuster. H-factors in dense graphs. J. Combin. Theory Ser. B, 66(2):269$282,1996$.

[2] F. Chung. Quasi-random hypergraphs revisited. Random Structures Algorithms, 40(1):39-48, 2012.

[3] F. R. K. Chung. Quasi-random classes of hypergraphs. Random Structures Algorithms, 1(4):363-382, 1990.

[4] F. R. K. Chung and R. L. Graham. Quasi-random hypergraphs. Random Structures Algorithms, 1(1):105-124, 1990.

[5] F. R. K. Chung and R. L. Graham. Quasi-random set systems. J. Amer. Math. Soc., 4(1):151-196, 1991.

[6] F. R. K. Chung and R. L. Graham. Cohomological aspects of hypergraphs. Trans. Amer. Math. Soc., 334(1):365-388, 1992.

[7] F. R. K. Chung, R. L. Graham, and R. M. Wilson. Quasi-random graphs. Combinatorica, 9(4):345-362, 1989.

[8] D. Conlon, H. Hàn, Y. Person, and M. Schacht. Weak quasi-randomness for uniform hypergraphs. Random Structures Algorithms, 40(1):1-38, 2012.

[9] A. Czygrinow, L. DeBiasio, and B. Nagle. Tiling 3-uniform hypergraphs with $k_{4}^{3}-2 e$. to appear in Journal of Graph Theory.

[10] D. Dellamonica, Jr. and V. Rödl. Hereditary quasirandom properties of hypergraphs. Combinatorica, 31(2):165-182, 2011.

[11] A. Hajnal and E. Szemerédi. Proof of a conjecture of P. Erdős. In Combinatorial theory and its applications, II (Proc. Colloq., Balatonfüred, 1969), pages 601-623. NorthHolland, Amsterdam, 1970. 
[12] H. Hàn, Y. Person, and M. Schacht. On perfect matchings in uniform hypergraphs with large minimum vertex degree. SIAM J. Discrete Math., 23(2):732-748, 2009.

[13] P. Keevash. The existence of designs. http://arxiv.org/abs/1401.3665.

[14] P. Keevash. A hypergraph blow-up lemma. Random Structures Algorithms, 39(3):275$376,2011$.

[15] P. Keevash and R. Mycroft. A geometric theory for hypergraph matching. to appear in Mem. Amer. Math. Soc.

[16] I. Khan. Perfect matchings in 4-uniform hypergraphs. arXiv:1101.5675.

[17] I. Khan. Perfect matchings in 3-uniform hypergraphs with large vertex degree. SIAM J. Discrete Math., 27(2):1021-1039, 2013.

[18] Y. Kohayakawa, B. Nagle, V. Rödl, and M. Schacht. Weak hypergraph regularity and linear hypergraphs. J. Combin. Theory Ser. B, 100(2):151-160, 2010.

[19] Y. Kohayakawa, V. Rödl, and J. Skokan. Hypergraphs, quasi-randomness, and conditions for regularity. J. Combin. Theory Ser. A, 97(2):307-352, 2002.

[20] J. Komlós, G. N. Sárközy, and E. Szemerédi. Blow-up lemma. Combinatorica, 17(1):109$123,1997$.

[21] J. Komlós, G. N. Sárközy, and E. Szemerédi. Proof of the Alon-Yuster conjecture. Discrete Math., 235(1-3):255-269, 2001. Combinatorics (Prague, 1998).

[22] D. Kühn and D. Osthus. Loose Hamilton cycles in 3-uniform hypergraphs of high minimum degree. J. Combin. Theory Ser. B, 96(6):767-821, 2006.

[23] D. Kühn and D. Osthus. The minimum degree threshold for perfect graph packings. Combinatorica, 29(1):65-107, 2009.

[24] D. Kühn, D. Osthus, and T. Townsend. Fractional and integer matchings in uniform hypergraphs. http://arxiv.org/abs/1304.6901.

[25] D. Kühn, D. Osthus, and A. Treglown. Matchings in 3-uniform hypergraphs. J. Combin. Theory Ser. B, 103(2):291-305, 2013.

[26] J. Lenz and D. Mubayi. Eigenvalues and linear quasirandom hypergraphs. submitted. http://arxiv.org/abs/1208.4863.

[27] J. Lenz and D. Mubayi. Eigenvalues of non-regular linear quasirandom hypergraphs. online at http://arxiv.org/abs/1309.3584.

[28] J. Lenz and D. Mubayi. Perfect packings in quasirandom hypergraphs. online at http://arxiv.org/abs/1402.0884. 
[29] J. Lenz and D. Mubayi. The poset of hypergraph quasirandomness. accepted in Random Structures and Algorithms. http://arxiv.org/abs/1208.5978.

[30] A. Lo and K. Markström. F-factors in hypergraphs via absorption. preprint arXiv:1105.3411.

[31] A. Lo and K. Markström. Minimum codegree threshold for $\left(K_{4}^{3}-e\right)$-factors. J. Combin. Theory Ser. A, 120(3):708-721, 2013.

[32] K. Markström and A. Ruciński. Perfect matchings (and Hamilton cycles) in hypergraphs with large degrees. European J. Combin., 32(5):677-687, 2011.

[33] O. Pikhurko. Perfect matchings and $K_{4}^{3}$-tilings in hypergraphs of large codegree. Graphs Combin., 24(4):391-404, 2008.

[34] V. Rödl, A. Ruciński, and E. Szemerédi. Perfect matchings in large uniform hypergraphs with large minimum collective degree. J. Combin. Theory Ser. A, 116(3):613-636, 2009.

[35] A. Shapira1 and R. Yuster. The quasi-randomness of hypergraph cut properties. Random Structures Algorithms, 40(1):105-131, 2012.

[36] A. Thomason. Pseudorandom graphs. In Random graphs '85 (Poznań, 1985), volume 144 of North-Holland Math. Stud., pages 307-331. North-Holland, Amsterdam, 1987.

[37] A. Thomason. Random graphs, strongly regular graphs and pseudorandom graphs. In Surveys in combinatorics 1987 (New Cross, 1987), volume 123 of London Math. Soc. Lecture Note Ser., pages 173-195. Cambridge Univ. Press, Cambridge, 1987.

[38] H. Towsner. Sigma-algebras for quasirandom hypergraphs. available online at http://arxiv.org/abs/1312.4882.

[39] A. Treglown and Y. Zhao. Exact minimum degree thresholds for perfect matchings in uniform hypergraphs. J. Combin. Theory Ser. A, 119(7):1500-1522, 2012.

[40] A. Treglown and Y. Zhao. Exact minimum degree thresholds for perfect matchings in uniform hypergraphs II. J. Combin. Theory Ser. A, 120(7):1463-1482, 2013. 